\title{
Análisis de la factura electrónica en el contexto de la Transformación Digital en la Unión Europea
}

\author{
Raúl Oltra Bádenes ${ }^{a}$, Vicente Guerola-Navarro ${ }^{\text {, }}$, Doina Stratuc, Juan V. Oltra \\ Gutiérrez $^{\text {, }}$, Hermenegildo Gil Gómez ${ }^{\mathrm{e}}$ \\ ${ }^{\mathrm{a}-\mathrm{e}}$ Departamento de Organización de Empesas, Universitat Politècnica de València, Valencia. \\ \{rauloltra@doe; viguena@omp; doistst@doctor; jvoltra@omp; hgilgom@omp\}.upv.es
}

\section{Resumen}

La adopción de un estándar de generación, comunicación e intercambio de factura electrónica es uno de los hitos de la Unión Europea (UE) dentro de los mecanismos de cohesión interterritorial. Su uso generalizado en los países Miembros debe favorecer el acceso efectivo de los ciudadanos a bienes y servicios ofrecidos por proveedores transfronterizos, lo cual debe redundar en un beneficio colectivo tanto por el ahorro en costes, tiempo, y materiales, como por la creación de un canal económica y medioambientalmente sostenible de intercambio de datos de facturación.

Dentro de los esfuerzos de la UE por crear redes transfronterizas de comunicación para eliminar fronteras a la libre circulación de bienes y servicios, el proyecto de facturación electrónica se ha llevado a cabo mediante varias acciones entre las que destaca el proyecto GOV2EU - Apoyo a entidades públicas para adoptar el Estándar de la UE sobre factura electrónica para transacciones transfronterizas. Este proyecto se ha llevado a cabo mediante la colaboración de entidades públicas y privadas, financiado con fondos públicos de la UE.

En este artículo estudiamos el contexto en el que se desarrolla el concepto y puesta en práctica de la factura electrónica, más en concreto a través del proyecto GOV2EU, partiendo de los componentes legislativos y técnicos necesarios para que la facturación electrónica sea una realidad. Las conclusiones muestran el éxito de la implementación de la comunicación efectiva entre las 15 entidades participantes (12 de ellas hospitales).

Se concluye pues que la facturación electrónica es una herramienta válida y viable entre entidades que proveen bienes y servicios de forma transfronteriza entre Estados Miembros de la UE. 
Palabras clave: Factura electrónica, e-Invoice, GOV2EU.

\section{Introducción}

En el entorno globalizado en el que nos encontramos actualmente, las tecnologías y los sistemas de información son un factor competitivo, que permiten a las organizaciones tener la información disponible en el momento y forma adecuados. De este modo, las organizaciones pueden tomar mejores decisiones, y ser más eficientes en su gestión. Es evidente que, cuanto antes se disponga de la información, antes se podrá tomar la decisión que dependa de ella, o esta se tomará con más elementos de juicio, y, por tanto, de forma más adecuada.

En este entorno, la facturación electrónica mejora notablemente los tiempos de gestión de las facturas entre dos organizaciones (bien sean públicas o privadas) y con ello, mejora su eficiencia y los tiempos de respuesta en todo el proceso de transacciones comerciales. Esto reducirá drásticamente la carga administrativa en términos de tiempo, gasto y esfuerzo asociados con el uso de servicios públicos extranjeros en nuestro mundo cada vez más globalizado (CEF Digital, 2018). Por este motivo, desde hace ya unos años, el marco de la Unión Europea ha impulsado el desarrollo e implantación de la factura electrónica los países miembros, financiando proyectos de desarrollo y estableciendo un marco de referencia para la facturación electrónica. De hecho, el programa "Connecting Europe Facility” (CEF) tiene una línea específica para el desarrollo e implantación de la factura electrónica en los países miembros de la UE, el denominado "CEF eInvoicing building block".

Por tanto, es indudable la importancia y la repercusión que la factura electrónica puede tener en la eficiencia de las organizaciones. Pero también en el entorno global, en el que, mediante la eliminación de documentos en papel, se dará un paso más en la búsqueda de la sostenibilidad en sus tres aspectos, no sólo en el económico, sino también en el social y el medioambiental.

Con ese objetivo surge el proyecto GOV2EU (CEF Telecom, 2016), que apoya a las entidades públicas a adoptar el estándar de la UE sobre facturas electrónicas para transacciones transfronterizas. Posteriormente se lanza, en la misma línea el proyecto HOSP\&INVOICE, mediante el cual hospitales públicos de Bélgica, Austria, Hungría e Italia se prepararon para la norma de facturación electrónica. 


\subsection{Proyecto GOV2EU para que entidades públicas}

Nos centramos en este estudio en el proyecto GOV2EU - Supporting public entitics to adopt EU standard on electronic invoice for cross-border transactions.

En este proyecto ha sido clave la participación de los proveedores de servicios de intercambios electrónicos de datos (EDI providers) que han sido representados en este proyecto por EDICOM dentro del consorcio creado para este efecto. Ha sido también clave la participación de INNOVATION AND NETWORKS EXECUTIVE AGENCY (INEA) como la agencia de que se ha encargado de la ejecución del proyecto. Y finalmente ha habido un tercer actor clave como Academia de gestión y dirección del proyecto, en este caso la Universitat Politécnica de Valencica (UPV).

El proyecto apoya la adopción de la plataforma de nube electrónica EDICOM B2B que cumple con el estándar europeo de facturación electrónica (EN) por parte de entidades públicas, a saber, 36 hospitales en Austria, Bélgica, Italia y Hungría. Esto permitirá que los hospitales públicos envíen y reciban facturas electrónicas que cumplan con la EN (compatibles con ambas sintaxis de EN OASIS UBL2.1 y UN / CEFACT CII).

Los hospitales públicos, con el apoyo de EDICOM B2B Cloud Platform, han desarrollado su software ERP (Enterprise Resource Planning) integrando el modelo semántico del estándar europeo de facturación electrónica. El desarrollo de ERP será seguido por el despliegue de la interoperación e intercomunicación automatizadas dentro de la plataforma B2B EDICOM utilizando su propio punto de acceso (AS2 y AS4).

El proyecto se ha basado en los resultados de las acciones financiadas anteriormente por CEF 2015-EU-IA-0058 y 2016-EU-IA-0096. El primero resultó en la actualización de las herramientas de facturación electrónica de la plataforma en la nube B2B EDICOM para cumplir con la Directiva de facturación electrónica (Directiva 2014/55 / UE) en el sector de la salud. Este último permitió que entidades adicionales del sector de la salud envíen y reciban Facturas electrónicas que cumplan con EN. Este proyecto reutiliza estas herramientas de facturación electrónica e las integra con los sistemas ERP de 36 hospitales de diferentes Estados miembros, utilizando recursos ya desplegados para reducir costos y amplificar el impacto en Europa.

El proyecto ha dado como resultado que 36 hospitales públicos (12 de Bélgica, 14 de Italia, 9 de Hungría y 1 de Austria) con el apoyo de EDICOM y ASSECO puedan intercambiar facturas electrónicas que cumplan con la EN (compatible con ambas sintaxis UBL 2.1 y CEFACT / CII) 


\section{Marco del Proyecto}

La iniciativa "Connecting Europe Facility" (CEF) se creó mediante el Reglamento (UE) n ${ }^{\circ}$ 1316/2013 del Parlamento Europeo y del Consejo (European Commision, 2013). Este Reglamento determina las condiciones, métodos y procedimientos para proporcionar asistencia financiera de la Unión a las redes transeuropeas a fin de apoyar proyectos de interés común, además de establecer el desglose de los recursos disponibles para el periodo 2014-2020 en los campos de transporte, telecomunicaciones y energía.

En este caso nos interesa la parte aplicable a la factura electrónica (CEF Telecom, 2019a). La facturación electrónica es el intercambio de un documento de factura electrónica entre un proveedor y un comprador. Una factura electrónica (eInvoice) es una factura que se ha emitido, transmitido y recibido en un formato de datos estructurados que permite su procesamiento automático y electrónico, tal como se define en la Directiva 2014/55 / UE (CEF Telecom, 2019b).

Como objetivo general figura la mejora de la infraestructura física y digital de nuestras redes facilitando, entre otros factores, el uso de infraestructuras interoperables para poder favorecer las transacciones y las consecuentes facturaciones. Con todo ello se debería favorecer el acceso de los ciudadanos europeos a bienes y servicios sin importar el país desde el que se provisionan.

\subsection{CEF eInvoicing building block}

El building block de la facturación electrónica de CEF, tiene como objetivo promover la adopción y acelerar el uso de la facturación electrónica con respecto al estándar europeo, tanto entre las entidades públicas y privadas establecidas en la UE, como en los países participantes del EEA (CEF Digital, 2019). Sirve para ayudar a las administraciones públicas a cumplir con la legislación de facturación electrónica de la UE, y ayuda a los proveedores de servicios y soluciones a adaptar sus servicios en consecuencia. Por lo tanto, el building block de facturación electrónica del CEF, se centra especialmente en el tipo de transacciones Business to Government (B2G), aunque también puede permitir la comunicación de gobierno a gobierno (G2G). Sin embargo, otro tipo de comunicaciones digitales, como Business to Business (B2B), Government to Citizen (G2C) o Business to Consumer (B2C) no se abordan en el contexto del building block de facturación electrónica. 


\subsection{La facturación Electrónica}

La facturación electrónica (CEF Digital, 2019a) es el intercambio de un documento de factura electrónica entre un proveedor y un comprador. Una factura electrónica (eInvoice) es una factura que se ha emitido, transmitido y recibido en un formato de datos estructurados que permite su procesamiento automático y electrónico, tal como se define en la Directiva 2014/55 / UE.

Una factura electrónica estructurada contiene datos del proveedor en un formato legible por máquina, que puede importarse automáticamente al sistema de Cuenta por pagar (AP) del comprador sin necesidad de ingresar manualmente.

Al comparar las facturas electrónicas con las facturas en papel, es útil tener en cuenta que las facturas en papel tienen tres características que están tan integradas que normalmente no nos damos cuenta de que pueden separarse. Las facturas en papel contienen detalles de datos, como los importes, descripciones y cantidades; proveen esos datos en un formato visual, en papel impreso, que puede leerse manualmente; y finalmente tienen una forma física que los permite ser manejadas e intercambiadas manualmente.

Las imágenes digitales (pdf y otras formas visuales digitales) de facturas eliminan el elemento físico y permiten que las facturas se manejen y archiven de una manera más eficiente que el papel. Sin embargo, estos formatos aún requieren que la factura se vea manualmente y que sus datos se lean e ingresen manualmente en los sistemas de información. Sin embargo, las facturas electrónicas solo contienen los datos en forma estructurada, y pueden importarse automáticamente a los sistemas de información, por lo que no incluyen una presentación visual de los datos de la factura, aunque se pueden procesar temporalmente durante el procesamiento o transponer a formatos visuales. Para las facturas electrónicas, el formato visual es secundario y el objetivo en la automatización no es ver la factura, excepto en casos irregulares. Evidentemente, sí que se puede crear una versión visual y legible por humanos de la factura electrónica, para fines de lectura y puede fluir dentro del mensaje estructurado, pero no se considera parte de la factura electrónica en sí misma.

De este modo, es importante tener claro que, pese a lo que en muchos entornos se piensa, las facturas electrónicas no son:

- Datos de factura no estructurados emitidos en formato pdf o Word.

- Imágenes de facturas como jpg de tiff.

- Facturas HTML no estructuradas en una página web o en un correo electrónico.

- OCR (facturas de papel escaneadas)

- Facturas en papel enviadas, como imágenes, a través de máquinas de fax

El uso de facturas electrónicas requiere dos funciones clave: 
- La factura electrónica debe crearse con la estructura correcta.

- La factura electrónica debe transferirse del sistema del vendedor al sistema del comprador.

Para ello, existe una norma europea sobre facturación electrónica, EN16931, que define la estructura de una factura electrónica y varias opciones para la transmisión de la factura entre los diferentes actores que la deben gestionar.

\subsection{Beneficios de la factura electrónica}

La adopción masiva de la facturación electrónica en la UE genera importantes beneficios económicos y un aumento de la competitividad empresarial europea. Con el respaldo de la legislación europea, la aceptación de las facturas electrónicas por parte de los gobiernos facilitará las actividades comerciales con el sector público (CEF Digital, 2019b).

Los beneficios de reemplazar las facturas en papel por facturas electrónicas se pueden alcanzar en dos etapas:

- Reemplazar el formulario en papel físico con un formulario digital permite que la factura se maneje y se archive de manera más eficiente. Esto proporciona importantes ahorros en impresión, franqueo, enrutamiento y archivado dentro de la oficina. Estos beneficios se pueden lograr con imágenes digitales de la factura, como pdf, jpg y html, así como con facturas electrónicas estructuradas.

- Hacer que los datos sean legibles por máquina elimina la necesidad de ver y leer manualmente una forma visual de la factura. También elimina el trabajo manual de ingresar la información de la factura en un sistema AP. Esto proporciona ahorros significativos en recursos humanos y reduce significativamente los errores en la entrada de datos. Esto no se puede lograr con imágenes digitales de la factura, como pdf.

La adopción de la facturación electrónica en el sector público puede generar beneficios:

- respalda las prioridades de las políticas públicas, como la reducción del déficit del sector público, la transparencia financiera y la promoción del desarrollo sostenible.

- específicamente hará una contribución importante a la reducción y eficiencia de costos del sector público.

- También proporcionará beneficios a los proveedores del sector privado y creará oportunidades para que el sector público actúe como un catalizador para la adopción más amplia de procesos digitales en común con el sector privado. 


\section{El Proyecto GOV2EU}

El proyecto GOV2EU nace de la necesidad de tener un sistema de facturación electrónica para las administraciones públicas, bajo las leyes europeas que cambian el proceso de facturación (UPV - ai2, 2019). Para hacer eso, el consorcio resolverá toda la complejidad relacionada con la intercomunicación y la integración de datos, coordinada por EDICOM, empleando la plataforma en la nube EDICOM B2B. Además, implementarán una solución de sintaxis múltiple capaz de manejar la facturación electrónica en múltiples estándares, de acuerdo con las necesidades de cada participante con sus propios sistemas ERP y las especificaciones detalladas en la regulación europea, para lograr documentos electrónicos semánticamente interoperables en El alcance de la UE.

El proyecto también se basa en las especificaciones PEPPOL considerando que todos los participantes utilizarán un punto de acceso Peppol para comunicarse con otros para intercambiar las facturas electrónicas.

\subsection{Objetivos}

El objetivo global de la acción era promover y acelerar la adopción de la facturación electrónica principalmente en el sector de la salud mediante la reutilización de las herramientas de facturación electrónica para la plataforma de nube B2B EDICOM que se desarrollaron como resultado de la anterior acción 2015-EU-IA-0058 hospitales y otras entidades del consorcio de diferentes Estados miembros, lo que permite el intercambio de facturas electrónicas de acuerdo con las especificaciones de interoperabilidad semántica establecidas en la Directiva de facturación electrónica 2014/55 / UE.

Los objetivos del proyecto GOV2EU son (GOV2EU - ai2, 2019):

- promover la aceptación y el uso de la factura electrónica

- facilitar a las empresas de la UE la facturación transfronteriza al NHS utilizando la facturación electrónica

- Respetar los requisitos legales europeos (Directiva 2014/55 / UE)

\subsection{Participantes}

En este proyecto participan 9 socios de 9 países diferentes: 4 universidades, 8 empresas privadas y 17 hospitales bajo la coordinación de EDICOM (proveedor de plataforma de facturación electrónica). En concreto, y por paises: 
- Belgium: Universitair Ziekenhuis Gent

- France: Hôpitaux Du Pays du Mont Blanc, Université de Besançon FrancheComte

- Germany: HBS, Alfried Krupp, EKBG, Witten

- Hungary: Asseco HU, Borbála, BPK, MFTH, Soproni, Baja, Uzsoki, Péterfy, Hatvan, Margit, OORI

- Italy: LIMA

- Poland: ILIM, Suwalki

- Portugal: SUCH, SISCONSULT

- Slovakia: Asseco SK, Comenius University, SKMBP,

- Spain: Universitat Politècnica de València (university); Valtiva, Edicom (enterprises)

\section{3. fases del proyecto}

El proyecto ha constado de varias actividades o fases, con sus respectivos hitos de control, todas ellas realizadas con éxito a fecha de finalización del proyecto el 28 de febrero de 2019.

\section{Actividad 1: Gestión de Proyecto. Finalizado 30/11/2017}

- Proyecto lanzado: Basado en el inicio de la reunión del proyecto, presentación de las actividades a los socios y plan de trabajo para cada actividad.

- Directrices de gestión de proyectos: las directrices de gestión de proyectos comunicadas a todos los beneficiarios.

Actividad 2: Requisitos técnicos y legales y apoyo a la implementación. Finalizado $\underline{31 / 12 / 2017 .}$

- Pautas de implementación de usuarios finales de salud pública: se prepararon las pautas técnicas para la implementación de facturación electrónica por parte de los usuarios finales beneficiarios.

- Inicio del despliegue técnico: análisis previo de requisitos técnicos y legales completado por cada participante.

Actividad 3: Implementaciones de usuarios finales de salud pública. Finalizado 28/02/2019

- Plan de implementación del usuario final de salud pública listo: el plan para implementar la solución e inscribir las pruebas de interoperabilidad estará listo para ser seguido por todos los usuarios participantes. 
- Plan de prueba de plataforma y usuario completado: definición de planes de prueba para plataformas operativas.

- Facturación electrónica integrada en los sistemas de usuarios finales: sistemas de facturación electrónica totalmente operativos para todos los usuarios finales.

- Comienzo de las pruebas de integración.

- Validación de las facturas electrónicas por parte de la Plataforma de Servicio Central: la plataforma de prueba GITB (de EC-DIGIT) se utiliza para evaluar el cumplimiento de una factura electrónica dada a EN, y se corrigen errores.

\section{Actividad 4: Plan de Comunicación, Difusión y Explotación.. Finalizado 28/02/2019}

- Plan de comunicación listo: el plan de comunicación que deben seguir todos los beneficiarios y el sitio web del proyecto.

- Actividades de comunicación realizadas por todos los participantes, presentando evidencias y resultados de difusión.

\section{Resultados Obtenidos}

\subsection{Principales resultados}

Como resultado del proyecto GOV2EU, resultante de la Action 2016-EU-IA-0096, se han obtenido los siguientes resultados:

1. Mejorar la interoperabilidad de la facturación electrónica, a través de la incorporación de herramientas de facturación electrónica en EDICOM B2B Cloud Platform, por entidades públicas y privadas principalmente en el sector de la salud (12 hospitales, otras 3 entidades del consorcio) para facilitar la adopción de la Directiva de facturación electrónica. La plataforma incluye una solución multisintaxis para mapear y convertir las facturas siguiendo ambas direcciones: remitente de la factura (de un archivo de integración o sintaxis nacional en UBL 2.1 y CII) y receptor de la factura (convertir facturas en formatos UBL 2.1 y CII en cualquier otro formato e integración archivos).

2. Aumentar la conciencia del usuario y la adopción de la Directiva de facturación electrónica.

3.

\subsection{Estado actual}

Con respecto al estado actual, la Acción se implementó completamente a tiempo para el 28 de febrero de 2019 y se alineó con el programa de facturación electrónica de CEF. Podría 
proporcionar la solución específica de facturación electrónica para cada hospital y otras entidades del consorcio, cada una de ellas pasando el correspondiente Servicio de pruebas de conformidad de facturación electrónica CEF (plataforma GITB OF EC-DIGIT), alcanzando el Hito 10 definido por el Acuerdo de subvención. La difusión de la acción fue relevante. Utilizamos diferentes enfoques de marketing para informar al público que los hospitales y otras entidades del consorcio GOV2EU habían adoptado la Norma Europea sobre la factura electrónica, y también difundieron la Directiva de facturación electrónica 2014/55 / EU y el componente básico de facturación electrónica CEF en Europa.

El estado actual confirma que el proyecto está completamente implementado, alcanzando todos los hitos (todos los medios de verificación y algunas pruebas adicionales se presentan en varios informes preparados por los participantes), y los usuarios finales (hospitales y laboratorios / entidades) están completamente preparados para intercambiar facturas electrónicas, de acuerdo con la norma europea:

- $\quad$ Los 12 hospitales del consorcio:

- Szent Borbála Kórház (Borbála), Hungría

○ Bugát Pál Kórház (BPK), Hungría

○ Markhot Ferenc Teaching Hospital and Outpatient Clinic (MFTH), Hungría

- Soproni Erzsébet Oktató Kórház és Rehabilitációs Intézet (Soproni), Hungría

- Baja Szent Rókus Kórház (Baja), Hungría

- Uzsoki Utcai Kórház (Uzsoki), Hungría

○ Péterfy Sándor utcai Kórház rendelőintézet és Baleseti Központ (Péterfy), Hungría

- Albert Schweitzer Kórház- Rendeloinezet (Hatvan), Hungría

- Szent Margit Kórház (Margit), Hungría

- Országos Orvosi Rehabilitációs Intézet (Instituto Nacional de Rehabilitación Médica) (OORI), Hungría

- Nemocnica svätého Michala (SKMBP), Eslovaquia

- Universitair Ziekenhuis Gent (UZ Gent), Bélgica

- $\quad$ Y otras 3 entidades:

- LIMACORPORATE SPA (LIMA), Italia

- Universite De Besancon Franche-Comte (UFC), Francia

- Serviço de Utilização Comum dos Hospitais (SUCH), Portugal 


\section{Conclusiones}

Al considerar tanto los objetivos como los resultados, la conclusión es que la implementación de la acción se logró por completo y todos los elementos de las actividades (actividades e hitos) se desarrollaron en el tiempo acordado establecido en el Acuerdo de lanzamiento de la acción. La implementación de eInvoice cumple con el estándar europeo, y las soluciones pasaron el servicio de pruebas de conformidad de la Comisión Europea (CEF eInvoicing Conformance Testing Service), validando los resultados alcanzados y haciendo que INEA / DIGIT valide el proyecto en todos sus hitos de control. Esta validación supone el logro de los objetivos principales de la acción, y el logro de tareas individuales y los objetivos generales del proyecto.

\section{Agradecimientos}

Los autores de la presente comunicación quieren agradecer expresamente a la Comisión Europea y a la Agencia Ejecutiva de Innovación y Redes (Innovation and Networks Executive Agency), así como, al programa marco de financiación Connecting Europe Facility (CEF Telecom) por la financiación otorgada dentro de este marco al proyecto 'Supporting public entities to adopt EU Standard on electronic invoice for cross-border transactions (GOV2EU) con el número de Acción 2016-EU-IA-0096 y número de Grant Agreement INEA/CEF/ICT/A2016/1333510.

\section{Referencias}

CEF Digital, 2018. What are the benefits of eID?. Obtenido de: https://ec.europa.eu/cefdigital/wiki/display/CEFDIGITAL/Benefits+of+eID

CEF Digital, 2019a. What is eInvoicing. Obtenido de: https://ec.europa.eu/cefdigital/wiki/display/CEFDIGITAL/What+is+eInvoicing

CEF Digital, 2019b. What are the benefits?. Obtenido de: https://ec.europa.eu/cefdigital/wiki/display/CEFDIGITAL/What+are+the+benefits+of+e Invoicing

CEF Telecom, 2016. GOV2EU - Supporting public entities to adopt EU Standard on electronic invoice for cross-border transactions. Obtenido de: https://ec.europa.eu/inea/en/connecting-europe-facility/cef-telecom/2016-eu-ia-0096 
CEF Telecom, 2019a. 2019 CEF Telecom Call - eInvoicing (CEF-TC-2019-1). Obtenido de: $\quad$ https://ec.europa.eu/inea/en/connecting-europe-facility/cef-telecom/applyfunding/2019-einvoicing

CEF Telecom, 2019b. CALL FOR PROPOSALS CONCERNING PROJECTS OF COMMON INTEREST UNDER THE CONNECTING EUROPE FACILITY IN THE FIELD OF TRANS-EUROPEAN TELECOMMUNICATION NETWORKS. Obtenido de: https://ec.europa.eu/inea/sites/inea/files/2019-1_einvoicing_call_text 0.1.pdf.

European Commision, 2013. CEF Telecom. Obtenido de: https://ec.europa.eu/inea/en/connecting-europe-facility/cef-telecom

GOV2EU - ai2, 2019. GOV2EU. Obtenido de: http://gov2eu.ai2.upv.es/

UPV - ai2, 2019. GOV2EU: Supporting public entities to adopt EU Standard on electronic invoice for cross-border transactions. Obtenido de: https://www.ai2.upv.es/gov2eusupporting-public-entities-to-adopt-eu-standard-on-electronic-invoice-for-cross-bordertransactions/ 